\title{
The effect of periorbital cooling on pain, edema and ecchymosis after rhinoplasty: a randomized, controlled, observer-blinded study*
}

\author{
Oguz Kayiran', Caglar Calli² \\ 'MS Baltalimani Bone D. Training and Research Hospital, Department of Plastic and Reconstructive Surgery, Istanbul, Turkey \\ 2Ekol ENT Hospital, Department of Ear, Nose and Throat, Izmir, Turkey
}

Rhinology 54: 32-37, 2016

DOI:10.4193/Rhino15.177

*Received for publication:

July 2, 2015

Accepted: October 1, 2015

\begin{abstract}
Introduction: Success and satisfactory results in rhinoplasty are established not only with flawless surgery but also with meticulous perioperative care. Pain stays at the centre of these circumstances. Besides, several contributing perioperative factors such as periorbital edema and ecchymosis play key role on the patients' comfort.

Methods: Septorhinoplasty was carried out in 50 patients between February and May 2014 under general anesthesia. Local anesthesia with lidocaine and adrenaline combination was done prior to incision. Following the procedure, silicone gel packs were applied. One periorbital region was cooled after surgery whereas the opposite site was left uncooled. Periorbital edema-ecchmosis and pain intensity were graded and noted 1 hour, 1 day, 3 days, 1 week and 1 month after surgery.

Results: Cold application seriously reduced postoperative edema and ecchymosis at the first week ( $p=0.001$ for the first 3 days and $p=0.006$ at first week). Pain was reduced with cooling not on the first hour ( $p>0.05$ ), but on the forthcoming days throughout the first week $(p<0.005)$. Operation time revealed that primary cases were carried out quicker than revisional surgery. Moreover, pain scores were found lower in primary cases than revisions, especially in the first 3 days. These finding were approved statistically.
\end{abstract}

Conclusion: Cooling of the periorbital region reduces edema and ecchymosis as well as pain; however 3 days of use is enough after rhinoplasty. One hour after surgery, cooling does not affect the pain but reduce edema and ecchymosis.

Key words: pain, edema, ecchymosis, cooling, rhinoplasty, sensation

\section{Introduction}

Septorhinoplasty is very common procedure in aesthetic surgery. Considering the operative sequence, osteotomy mainstays at the critical step of rhinoplasty which creates variable amount of postoperative edema, ecchymosis and pain.

Cold application in rhinoplasty is mainly used to reduce the postoperative edema and ecchymosis, and pain. Despite its widespread use, therapeutic effects have not been fully elucidated (1). Little data exists demonstrating the effects of cooling on rhinoplasty statistically. However, there are several studies focusing on edema-ecchymosis reduction with steroid administration. Indeed, numerous combinations can be found such as single, separate, local or systemic use of steroids, even perioperatively, preoperatively or postoperatively ${ }^{(2-7)}$. The most preferred cooling system is the application of ice on the treated area. Another cooling option, silicone gel packs have the advantageous of the ease of application and 30 minutes cold duration. In addition, the packs can be easily provided and applied. More complicated cooling system, Hilotherapy, can also be chosen, especially in maxillofacial patients ${ }^{(1)}$. However, this system is not available in 
all facilities.

Pain is a subjective sensation which annoys the patient sometimes more than the procedure. The extent of pain is dependable upon many factors contributing upon each step of the premedication, surgical technique, postoperative period, drugs used and patient's sensation.

In the pain assessment, Visual Analog Scale (VAS) is the most frequent preferred tool in the post-operative period. This scale consists of a line extending between 0 (no pain) and 100 (unbearable pain). Verbal Rating Scale (VRS), ranging from a four-point list (no pain-mild pain-moderate pain-severe pain) can also be used to rate pain sensation via disabling affective psychological pain aspects ${ }^{(8)}$. Considering the 'analgesic ladder', non-steroid anti-inflammatory drugs (NSAID) are the most preferred agents worldwide, especially for the patients with a VAS score between 0-29. For the patients with VAS pain scores between 30-59, weak opioids (e.g. codeine, tramadol) are used. Patients in which these drugs fail and/or with a VAS score over 60 shall be given strong opioids (e.g fentanyl, morphine). Moreover, patient controlled anesthesia (PCA) allows more stabile blood level of the agent, intravenously ${ }^{(9-11)}$.

The objective of this study is to evaluate the effect of silicone gel packs applied postoperatively in septorhinoplasty patients. To us, this is the first prospective, randomized trial evaluating proposed effects considering periorbital cooling after rhinoplasty. In addition, cooling and uncooling were assessed at the same patient simultaneously.

\section{Patients and methods}

We conducted an observer-blinded, randomized, controlled trial in patients where septorhinoplasty was performed between February and May 2014. This study was approved by the ethics committee of the University Medical Center of Izmir (Trial Register IAEAHKN: 193) and informed consent was obtained from the entire of the patients. Patients in whom conchal or rib cartilages were used were excluded from the study. In addition, another exclusion criterion was the history of systemic diseases such as diabetes mellitus, hypertension, hematologic disorders etc. The entire of the surgeries were carried out by two authors.

\section{Surgery}

Single dosage Cefaclor ${ }^{(12)}$ was administered prior to surgery. $2 \%$ lidocaine with 1:100,000 adrenaline was used intra-operatively prior to incisions. Either open or closed approach was preferred; subperichondrial and subperiosteal tunnels were created in the operation to reduce the blood loss and the postoperative edema and ecchymosis ${ }^{(13)}$.

Open approach consisted as follows: 1) transcolumellar and infracartilaginous incision, 2) subperichondrial and subperiosteal tunnel creation, 3) septoplasty, 4) upper lateral cartilage
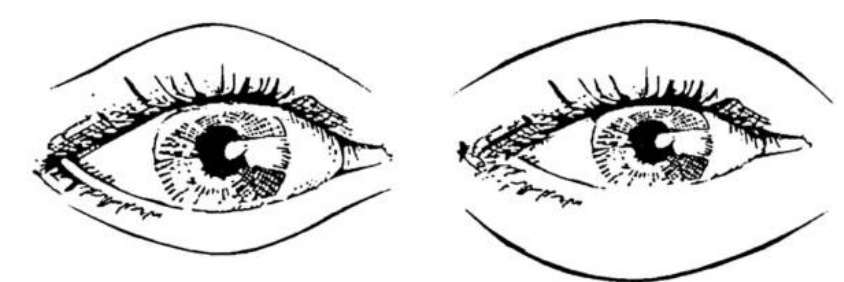

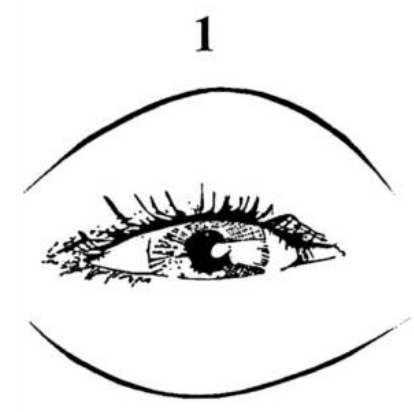

3

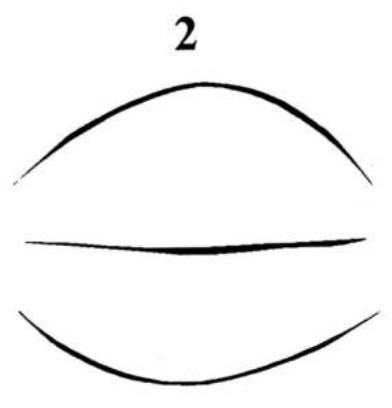

4
Figure 1. Scoring diagram for edema. Grade 1, no coverage of iris with eyelids; grade 2, slight coverage of iris with swollen eyelids; grade 3, full coverage of iris with swollen eyelids; grade 4, full closure of eyes.

reconstruction with fold-in flap technique $\left.{ }^{(14)}, 5\right)$ median, medial and/or lateral internal osteotomies, 6) tip rhinoplasty w/o local cartilage grafts, 7) closure and dressing.

Closed technique sequence was as: 1 ) transfixion and intracartilaginous incision, 2) subperichondrial and subperiosteal tunnel creation, 3) septoplasty, 4) upper lateral cartilage reconstruction, 5) median, medial and/or lateral internal osteotomies, 6) tip rhinoplasty, 7) closure and dressing. Osteotomies were carried out with guided median, Cinelli and guided-curved 4-mm lateral osteotomes.

Silicone gel packs were applied immediately after the extubation on one periorbital region for 20 minutes with 40 minutes intervals, 15 minutes with 45 minutes intervals and 10 minutes with 50 minutes intervals in the first, second, and third 24 hours, respectively. The other periorbital region was left uncooled. On the postoperative period, analgesic agents were not used until patients' referral. When needed, NSAIDs were the first choice; opioids were preferred if NSAIDs were unable to control the pain. Rupatadine was routinely used to prevent sneezing twicea-day for 3 days. Postoperative nausea and/or vomiting were controlled with the administration of $2 \mathrm{mg}$ ondansetron, when needed. Internal nasal packing was removed on the 3rd postoperative day and external cast on the 7th day. Periorbital edema and ecchymosis were graded and noted 1 hour, 1 day, 3 days, 1 week and 1 month after surgery according to the classification defined by Yucel (Figures 1 and 2) ${ }^{(15)}$. The blinded first author (OK) scored the edema and ecchymosis rates of the cooled and uncooled periorbital regions separately and randomly. 


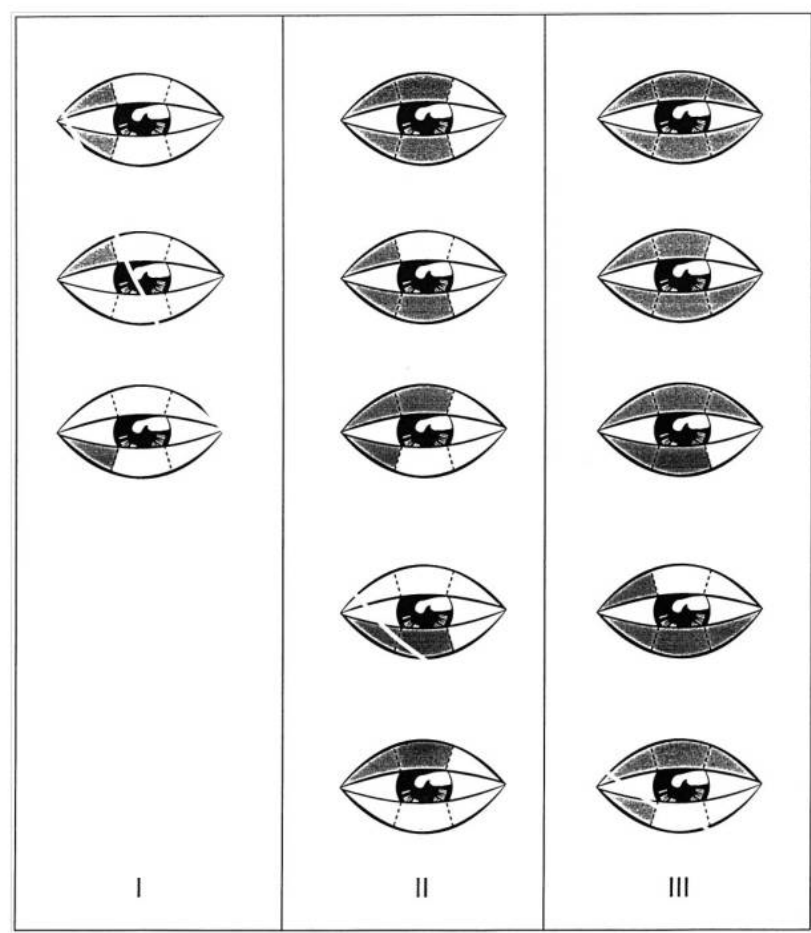

Figure 2. Scoring diagram for ecchymosis. Grade 1, ecchymosis up to the medial one-third part of the lower and/or upper eyelid; grade 2, ecchymosis up to the medial two thirds part of the lower and/or upper eyelid; grade 3, ecchymosis up to the full length of the lower and/or upper eyelid.

Each patient was asked to grade their present nasal pain intensity on a four-point verbal rating scale (VRS; no pain numbered as I, mild pain as II, mid pain as III or severe pain as IV) and visual analogue scale (VAS; 0-100 mm). A VAS score of 0 to 29 was entitled as $A, 30$ to 59 as B, and 60 to 100 as C. Patients recorded their VAS and VRS scores 1 hour, 1 day, 3 days, 1 week and 1 month after surgery. One month after surgery, patients were asked if they would have preferred cooling after rhinoplasty.

\section{Statistical analysis}

Data analysis was performed using SPSS statistical software ver. 20 for Windows (SPSS, Inc., Chicago, IL, USA). KolmogorovSmirnov test was used to evaluate whether the distribution of variables were normal. One-Way Analysis of Variance (ANOVA) was used for the comparisons of the operation time versus VAS and VRS scores and operation time versus edema and ecchymosis scores among two groups. Wilcoxon signed rank test was used to analyze whether the scores of pain, edema and ecchymosis were significantly different between the cooled and uncooled periorbital region at different time intervals. Chisquare test was preferred to compare the relationship between primary-revision and edema-ecchymosis rate. $P$ values lower than 0.05 were accepted as significant.

\section{Results}

Fifty patients were operated with a $34(68 \%)$ to $16(32 \%)$ female to male ratio. No complications such as infection, hematoma, bleeding were noted. Two patients were re-operated for functional reasons with a successful result. Mean age was 30.18 (Standard Deviation (SD): 9.89). Open techniques was used in 39 patients where closed techniques were preferred in 11 patients. Primary septorhinoplasty was carried out in 35 cases (70\%) whereas 15 were revision (30\%). Local cartilage grafts were used in 18 patients (36\%). Mean operation time was 67.74 minutes (SD: 18.55). 2 patients (4\%) requested painkillers.

\section{Edema and ecchymosis}

The scores for edema for the first week were found lower in cooled areas when compared to the uncooled areas $(p=0.001$ for the first hour, first and third days, $p=0.006$ for the first week). On the contrary, the scores for eccymosis showed no difference in the first postoperative hour $(p=0.071)$ and after first week $(p=0.103)$. However, one day after the operation, ecchymosis was significantly reduced on the cooled area until the third day ( $p=0.001$ for the first day and $p=0.004$ for the third day) (Figure 3).

\section{Pain}

Pain scores (VAS and VRS) noted by the patients was distributed and processed (Figure 4). One hour after surgery, there was not a significant difference on pain intensity of the cooled and uncooled areas ( $p=0.705$ for VAS, $p=0.439$ for VRS). However, one day after surgery, there was a significant drop on the cooled areas ( $p=0.001$ for VAS, $p=0.003$ for VRS) and this effect lasted until the third day ( $p=0.025$ for VAS, $p=0.014$ for VRS). After one week, no difference was found on pain perception $(p=0.257$ for VAS, $p=0.317$ for VRS).

\section{Other parameters}

Two patients requested painkiller administration in which NSAIDs were likely used. Age of the patient did not influence the edema or ecchmyosis scores ( $p>0.05$ ). Edema or ecchymosis scores were not related with the procedure whether it was primary or revision ( $p>0.05$ ). There was not a significant difference between the edema-ecchymosis scores and pain sensation, and this outcome was proven both for VAS and VRS scores ( $p>0.05$ ). Operation time was significantly a distinctive factor in primary or revision septorhinoplasty cases where primary cases were carried out quicker than revisions. Moreover, pain scores (VAS and VRS) throughout the first three postoperative days were found dependable with the operation time. Less operation time ensured less pain throughout the first three days postoperatively $(p<0.05)$. However, after the third day, operation time did not affect the pain.

All patients indicated that they would prefer cooling in order to 


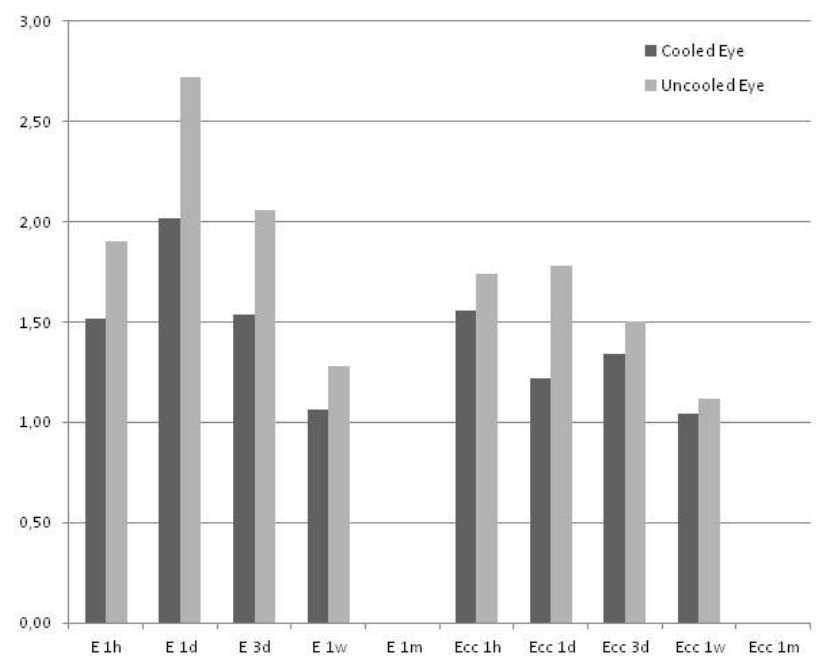

Figure 3. Mean edema and ecchymosis scores for the cooled and uncooled eyes (E indacates edema, Ecc indicates Ecchymosis; $1 \mathrm{~h}$ represents first postoperative hour, $1 \mathrm{~d}$ represents first postoperative day, $3 \mathrm{~d}$ represents third postoperative day $1 \mathrm{w}$ represents first postoperative week, $1 \mathrm{~m}$ represents first postoperative month).

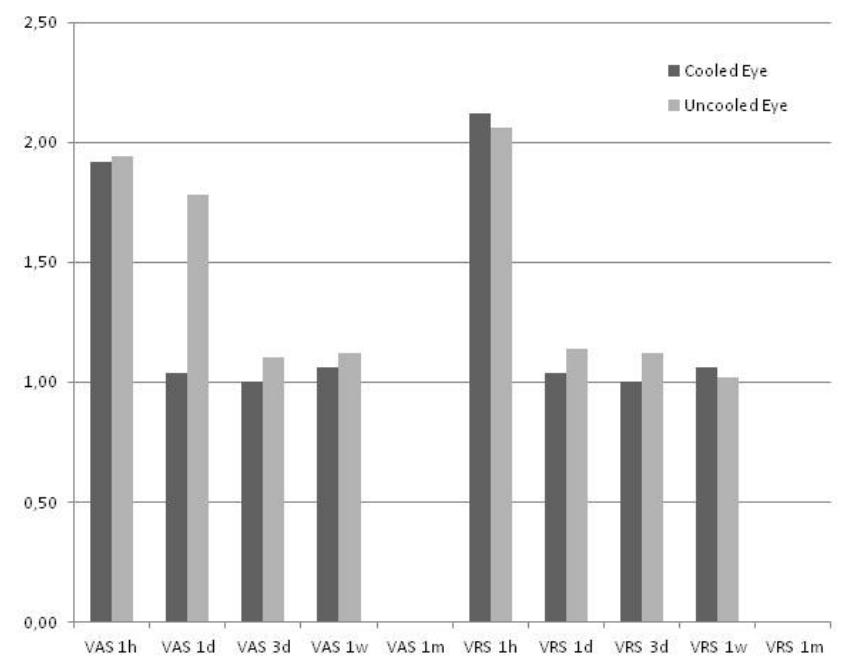

Figure 4. Mean pain scores for the cooled and uncooled eyes (VAS: Visual Analog Scale, VRS: Verbal Rating Scale, $1 \mathrm{~h}$ represents first postoperative hour, $1 \mathrm{~d}$ represents first postoperative day, $3 \mathrm{~d}$ represents third postoperative day $1 \mathrm{w}$ represents first postoperative week, $1 \mathrm{~m}$ represents first postoperative month). reduce the pain-edema-ecchymosis.

\section{Discussion}

On common belief and practice, the practitioners prefer cooling after rhinoplasty. The benefit of periorbital cooling after rhinoplasty is well-known for its widespread use. Oddly enough, there exists only one study regarding the effects of cooling on the reduction of edema and hematoma. Previous studies have shown that use of steroid and/or adrenaline and lidocaine combination diminishes the eyelid edema, ecchymosis and pain $(2-7,16,17)$. On the contrary, a detailed literature search with steroid use in cosmetic plastic surgery revealed no need for steroid administration in rhinoplasty in reducing postoperative edema and ecchymosis ${ }^{(18)}$. In a recent study, it was found that the addition of a single dose of pregabalin and dexamethasone to multimodal analgesia in rhinoplasty surgery provided efficient analgesia and thus decreased opioid consumption ${ }^{(16)}$. Regarding the mechanism of edema and eccyhmosis, the effect of local heparinoids was assessed in a study but it was not found effective in the prevention after rhinoplasty ${ }^{(19)}$. A recent study revealed the beneficial effects of tranexamic acid and corticosteroid combination in septorhinoplasty ${ }^{(20)}$.

Here, we conducted a prospective study demonstrating the effect of periorbital cooling on edema-ecchymosis-pain scores. These scores were analyzed separately and statistically. Moreover, we tried to establish a relationship among the operation time and pain, gender and pain, age and pain, age and edemaecchymosis, primary/revisional surgery and edema-ecchymosis. To us, this is the first study clarifying abovementioned parame- ters with each others.

Considering cold biologic effects, vasoconstriction, either locally or globally, affects the tissue. Pain reduction, cell metabolism decrement, immediate local vasoconstriction, decrease of muscular spasticity ${ }^{(21)}$ comprises the physiological responses of therapeutic cooling. Below $15^{\circ} \mathrm{C}$, paradox vasodilatation occurs due to paralysis of vascular smooth muscle ${ }^{(1)}$. In therapeutic use, cold has an anti-edema effect minimizing blood leakage. The mechanism of pain reduction is caused by the alteration of nerve endings and the conduction of pain impulses (21). Since ice application over the bare skin can facilitate skin necrosis, silicone gel packs were preferred. They have the advantage of 30 minute cold duration. Mean temperature for the packs is $+4^{\circ} \mathrm{C}$ and can be cooled down to $0^{\circ} \mathrm{C}$. The duration of cold effect was enough for the patient as well as the study. In a recent stu$d y$, edema and ecchymosis were found limited with the use of ice cooled swabs ${ }^{(22)}$. However, this practical application was not approved statistically. They proposed that this cooling was likely to facilitate a better cosmetic result. A recent study surprisingly revealed that cooling of eyelids after upper blepharoplasty did not reduce edema, erythema or hematoma, but reduced pain 1 day after surgery ${ }^{(23)}$. Here, we tried to establish more objective parameters concerning the effects of cooling and uncooling at the same patient, at the same time.

Lots of factors contribute in the consistence of pain ${ }^{(9,24-27)}$. In pain assessment, unidimensional and/or multidimensional methods can be chosen. Unidimensional pain questionnaires such as VAS and VRS solely rate sensory components of pain and omit affective psychological pain aspects ${ }^{(8,26)}$. In the postope- 
rative period, a VAS score between 0 and 29 indicates analgesic success, whereas scores above 70 immediate medication ${ }^{(10)}$. VRS questionnaires shall be added to assess the pain severity more objectively. Loos et al concluded that VRS should be favored over the VAS in postherniorraphy pain assessment ${ }^{(8)}$. Early postoperative pain assessment with VAS following septorhinoplasty was summarized in a study in which analgesia was recommended for all patients in the first postoperative 3 days ${ }^{(9)}$. According to this study, VAS scores were found elevated especially in the first evening after the operation. Here, we assessed both VAS and VRS in order to evaluate the pain intensity more objective. In pain management, NSAIDs were the first choice where opioids should be chosen in severe pain later on. In another study, PCA with lornoxicam vs. dipyrone were evaluated after septorhinoplasty where lornoxicam was found superior ${ }^{(27)}$. Our findings indicated that pain scores did not elevate one hour later postoperatively whereas one day later, pain was found higher significantly on uncooled areas. We recommend cooling the periorbital region after rhinoplasty instead of medication. Tebbet proposed that preference of atraumatic techniques will cause less edema and ecchymosis ${ }^{(28)}$. To us, subperichondrial and subperiosteal elevation of the nasal flap will cause less trauma and ensure reduced periorbital edema and ecchymosis in the postoperative period.

The satisfactory result in rhinoplasty is more dependable upon the shape of the nose obtained afterwards. Thus, until the external cast removal on the 7th postoperative day, neither the practitioner nor the patient could be able to analyze the shape of the nose, whereas the functional results could be assessed globally after the 3rd day. Therefore, to us, the first postoperative week and maintenance play the key part in the patient satisfaction. We believe that rhinoplasty in patients handled below $90 \mathrm{~min}$ need less medication, less hospitalization. Therapeutic cooling can be safely used in all of the patients with great orientation and cooperation to diminish pain and control edema-ecchymosis after rhinoplasty.

\section{Authorship contribution}

Study conception and design: OK, CC; Acquisition of data: OK; Analysis and interpretation of data: OK; Drafting of manuscript: OK, CC; Critical revision: OK, CC.

\section{Conflict of interest}

None declared.

\section{References}

1. Belli E, Rendine G, Mazzone N. Cold therapy in maxillofacial surgery. J Craniofac Surg. 2009;20(3):878-80.

2. Kara CO, Gökalan I. Effects of single-dose steroid usage on edema, ecchymosis, and intraoperative bleeding in rhinoplasty. Plast Reconstr Surg. 1999;104(7):2213-8

3. Gurlek A, Fariz A, Aydogan H, ErsozOzturk A, Eren AT. Effects of different corticosteroids on edema and ecchymosis in open rhinoplasty. Aesthetic Plast Surg 2006:30(2):150-4.

4. Assimes TL, Lessard ML. The use of perioperative corticosteroids in craniomaxillofacial surgery. Plast Reconstr Surg. 1999;103(1):313-21.

5. Berinstein TH, Bane SM, Cupp CL, DeMarco JK, Hunsaker DH. Steroid use in rhinoplasty: an objective assessment of postoperative edema. Ear Nose Throat J. 1998;77(1):40-3.

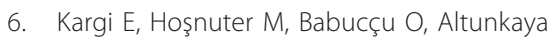
$\mathrm{H}$, Altinyazar C. Effect of steroids on edema, ecchymosis, and intraoperative bleeding in rhinoplasty. Ann Plast Surg. 2003;51(6):5704.

7. Gürlek A, Fariz A, Aydoğan H, Ersöz-Oztürk A, Evans GR. Effects of high dose corticosteroids in open rhinoplasty. J Plast Reconstr Aesthet Surg. 2009;62(5):650-5

8. Loos MJ, Houterman S, Scheltinga MR Roumen RM. Evaluating postherniorrhaphy groin pain: Visual Analogue or Verbal Rating Scale? Hernia. 2008;12(2):147-51.

9. Szychta P, Antoszewski B. Assessment of early post operative pain follow- ing septorhinoplasty. J Laryngol Otol. 2010;124(11):1194-9

10. Mantha S, Thisted R, Foss J, Ellis JE, Roizen MF. A proposal to use confidence intervals for visual analog scale data for pain measurement to determine clinical significance. Anesth Analg. 1993;77(5):1041-7.

11. Wewers ME, Lowe NK. A critical review of visual analogue scales in the measurement of clinical phenomena. Res Nurs Health. 1990;13(4):227-36.

12. Rajan GP, Fergie $N$, Fischer $U$, Romer $M$, Radivojevic V, Hee GK. Antibiotic prophylaxis in septorhinoplasty? A prospective, randomized study. Plast Reconstr Surg. 2005;116(7):1995-8.

13. Kara CO, Kara IG, Topuz B. Does creating a subperiosteal tunnel influence the periorbital edema and ecchymosis in rhinoplasty? J Oral Maxillofac Surg. 2005;63(8):1088-90.

14. Ozmen S, Ayhan S, Findikcioglu K, Kandal S, Atabay K. Upper lateral cartilage fold-in flap: a combined spreader and/or splay graft effect without cartilage grafts. Ann Plast Surg. 2008;61(5):527-32.

15. Yücel OT. Which type of osteotomy for edema and ecchymosis: external or internal? Ann Plast Surg. 2005;55(6):587-90.

16. Demirhan A, Tekelioglu UY, Akkaya A, Bilgi M, Apuhan T, Karabekmez FE, Bayir H, Kurt $A D$, Kocoglu H. Effect of Pregabalin and Dexamethasone Addition to Multimodal Analgesia on Postoperative Analgesia Following Rhinoplasty Surgery. Aesthetic Plast Surg. 2013 Sep 21.

17. Gun R, Yorgancılar E, Yıldırım M, Bakır S,
Topcu I, Akkus Z.Effects of lidocaine and adrenaline combination on postoperative edema and ecchymosis in rhinoplasty. Int J Oral Maxillofac Surg. 20111;40(7):722-9.

18. Pulikkottil BJ, Dauwe P, Daniali L, Rohrich RJ. Corticosteroid use in cosmetic plastic surgery. Plast Reconstr Surg. 2013;132(3):352e$60 \mathrm{e}$

19. Kelleş M, Erdem T, Fırat $Y$, Kalcıoğlu MT, Akarçay M, Selimoğlu E, Yoloğlu S. Efficacy of local heparinoids on preventing edema and ecchymosis after rhinoplasty. Kulak Burun Bogaz Ihtis Derg. 2010;20(4):191-4

20. Sakallioğlu Ö, Polat C, Soylu E, Düzer S, Orhan I, Akyiğit A.The efficacy of tranexamic acid and corticosteroid on edema and ecchymosis in septorhinoplasty. Ann Plast Surg. 2015;74(4):392-6

21. Guyton AC: Medical Physiology. Philedelphia, PA: WB Saunders Co, 1991.

22. Hettige R, Mansell N. Limiting oedema, ecchymosis and haemorrhage in septorhinoplasty with ice cooled swabs. Ann R Coll Surg Engl. 2014;96(5):395-6.

23. Pool SM, van Exsel DC, Melenhorst WB, Cromheecke $M$, van der Lei B. The effect of eyelid cooling on pain, edema, erythema, and hematoma after upper blepharoplasty: a randomized, controlled, observer-blinded evaluation study. Plast Reconstr Surg. 2015;135(2):277e-81e

24. Pavlin DJ, Chen C, Penaloza DA, Polissar NL, Buckley FP. Pain as a factor complicating recovery and discharge after ambulatory surgery. Anesth Analg. 2002;95(3):627-34.

25. Carr DB, Goudas LC. Acute pain. Lancet. 
1999:353(9169):2051-8.

26. Briggs M, Closs JS. A descriptive study of the use of visual analogue scales and verbal rating scales for the assessment of postoperative pain in orthopedic patients. J Pain Symptom Manage. 1999;18(6):438-46.

27. Sener M, Yilmazer C, Yilmaz I, Caliskan E, Donmez A, Arslan G. Patient-controlled analgesia with lornoxicam vs. dipyrone for acute postoperative pain relief after septorhinoplasty: a prospective, randomized, double-blind, placebo-controlled study. Eur J Anaesthesiol. 2008;25(3):177-82

28. Tebbet JB: Primary Rhinoplasty. St Louis, MO, Mosby, 2000, p 85
Oguz Kayiran, MD

MS Baltalimani Bone D. Training and

Research Hospital

Department of Plastic and Recons-

tructive Surgery

Istanbul

Turkey

E-mail:droguzk@yahoo.com 